\title{
BERGENIA CILIATA: ISOLATION OF ACTIVE FLAVONOIDS, GC-MS ANALYSIS, ADME STUDY AND INHIBITION ACTIVITY OF OXALATE SYNTHESIZING ENZYMES
}

\author{
SHWETA R. GOPHANE*, SAGAR R. JADHAO, PREETI B. JAMDHADE
}

School of Life Sciences, Swami Ramanand Teerth Marathwada University, Nanded 431606 India

Email: shweta.gophane@gmail.com

Received: 20 May 2021, Revised and Accepted: 14 Sep 2021

\section{ABSTRACT}

Objective: Bergenia ciliata (family-Saxifragaceae) is a well-known herb for kidney stone. The main objective of the study was the identification of flavonoids along with ADME profile. Another supportive objective was to check inhibition of enzymes which perform active role in oxalate synthesis.

Methods: The hydromethanolic extract was fractionated by liquid-liquid extraction to obtain ethyl acetate and ethyl ether fractions. The chemical structures of the purified compounds were identified by gas chromatography-mass spectrometry.

Results: A total of 12 volatile chemical compounds belonging to hydrocarbons, esters, alcohols, fatty acids, ketones, etc. were identified and characterized in ethyl acetate fraction through GC-MS analysis Fractions enriched in flavonoids showed glycolate oxidase and lactate dehydrogenase enzyme inhibition with $\mathrm{IC}_{50}$ value $(\mu \mathrm{g} / \mathrm{ml}) 65.76$ and 69.84 respectively. The kinetic behaviour of the extracts that inhibit the Glycolate oxidase and Lactate dehydrogenase activity was determined by the Lineweaver-Burk plot. The mode of inhibition of the studied plant extract was type of a non-competitive inhibition. ADMET screening of compounds successfully passed all the parameters of screening.

Conclusion: On the basis of the results, it was found that Bergenia ciliata (rhizome) may serve as a novel and rich source of therapeutic compounds and it can be further explored for urolithiasis treatment purposes.

Keywords: Flavonoids, GC-MS, ADME, Glycolate oxidase, Lactate dehydrogenase, Inhibition

(C) 2021 The Authors. Published by Innovare Academic Sciences Pvt Ltd. This is an open-access article under the CC BY license (https://creativecommons.org/licenses/by/4.0/] DOI: https://dx.doi.org/10.22159/ijpps.2021v13i11.42127. Journal homepage: https://innovareacademics.in/journals/index.php/ijpps.

\section{INTRODUCTION}

Flavonols are a class of flavonoids that have the 3-hydroxyflavone backbone (IUPAC name: 3-hydroxy-2-phenylchromen-4-one). Flavonoids help regulate cellular activity and fight off free radicals that cause oxidative stress on your body. In simpler terms, they help your body function more efficiently while protecting it against everyday toxins and stressors. Flavonoids are also powerful antioxidant agents. Flavonoid-rich foods, based on their surprising health effects, are well described as superfoods. These include all plant-origin foods, mainly tea, fruit, vegetables, grains, legumes, nuts, and wine [1]. Flavonoids are important for human health because of their antioxidant, antibacterial, antiviral, antihepatotoxic, antiosteoporotic, antiulcer, immunomodulatory, antiproliferative and apoptotic activity and anti-inflammatory activities and because they act as free radical scavengers as they are potential reducing agents that protect from oxidative damage [2-10]. Bergenia ciliata Sternb. (family-Saxifragaceae), a high-value plant of the Sikkim Himalaya has been investigated for antioxidant, antiurolithiac activity and bioactive compounds. However, scientific exploration of $B$. ciliata for phytochemicals and pharmacological properties is in infancy. With this view, the present study was undertaken to investigate $B$. ciliata rhizome ethanolic extract for antiurolithiac activity and bioactive compounds. Glycolate oxidase (GOX, EC 1.1.3.15) the key enzyme involved in oxalate synthesis. It was first associated with the disease primary hyperoxaluria type1 (PH1). The inhibition of GOX activity is a suitable therapeutic strategy for decreasing endogenous oxalate synthesis. The oxidation of Glycolate to glyoxylate is catalyzed by Glycolate oxidase [11] and the reduction of glyoxylate to Glycolate by lactate dehydrogenase (LDH) (EC 1.1.1.27) [12-14]. In normal pathway, alanine glyoxylate aminotransferase (AGT) converts glyoxylate to glycine and so prevents the conversion of glyoxylate to oxalate which further form complex with calcium to lead into the formation of calcium oxalate stones (fig. 1.) [15]. In the case of AGT deficiency, glyoxylate gets directly converted to oxalate by enzymes GOX and LDH. Hence their inhibition proves potential in the management of urolithiasis [16]. GC-MS analysis can identify pure compounds present at less than 1 gm [17]. Simple, cost-effective spectroscopic (UV-Vis, FTIR, GC-MS) methods together or separate can be used for detecting photo components in this sense as well as conventional methods [18-20]. So far reports on the systematic evaluation and scientific investigation of Bergenia ciliata or their phytoconstituents as glycolate oxidase and lactate dehydrogenase inhibitors are scare. The mode of interaction of extracted flavonoids (inhibitor) with enzyme, including identifying constituent compounds using GCMS, determining IC50 values using inhibition kinetics analysis and determining inhibitory patterns using Lineweaver-Burk plots and computer analysis using ADME were well described using this approach.

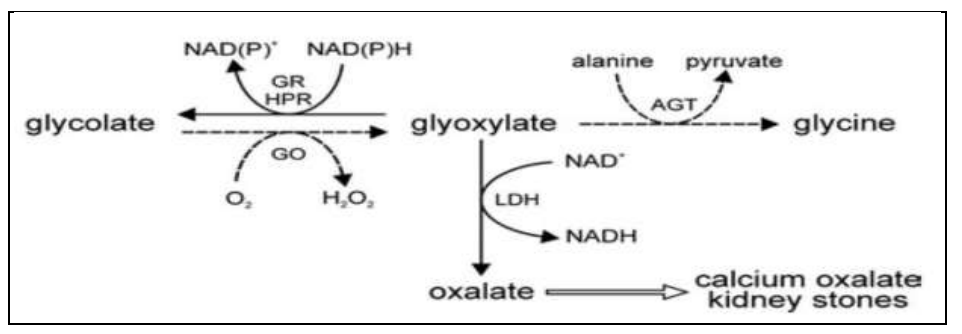

Fig. 1: Pathway associated with oxalate synthesis 


\section{MATERIALS AND METHODS}

\section{Flavonoid extraction from Bergenia ciliata (rhizome)}

Bergenia ciliata (rhizome) was procured from Yogesh pharma Pvt. Ltd., Nanded (MS), India. The plant material was washed thoroughly with water to remove dust and dried under the shade at room temperature for $5 \mathrm{~d}$. The dried parts were ground using blender to obtain the course powder and kept in an air-tight container till further use. Extraction of flavonoids was done as per method reported by Subramnian S and Nagarjan S [21]. Hundred grams of finely powdered sample were soxhlet extracted with $80 \%$ hot methanol $(500 \mathrm{ml})$ on a water bath for $24 \mathrm{~h}$ and filtered. The filtrate obtained was re-extracted successively with petroleum ether (fraction I), ethyl ether (fraction II), and ethyl acetate (fraction III) using separating funnel. Fraction of petroleum ether was discarded due to being rich in fatty substances. The fractions of (ethyl etherfraction II) and (ethyl acetate-fraction III) were further analysed for free and bound flavonoids, respectively. Ethyl acetate fraction of the sample was refluxed for the hydrolysis using $7 \% \mathrm{H}_{2} \mathrm{SO}_{4}$ for $2 \mathrm{~h}$ (for removal of bounded sugars) and again filtrate was extracted in separating funnel with ethyl acetate. Ethyl acetate extract thus obtained was washed with distilled water to neutrality. Ethyl ether (free flavonoids) and ethyl acetate fractions (bound flavonoids) were dried in rota vapour and weighed.

\section{Gas chromatography-mass spectrometry analysis}

The GC-MS analysis of the ethanolic extract was carried out using a Agilent 7890 A gas chromatogram equipped and coupled to a mass detector 5975 MSD spectrometer with DB 5 MS and $30 \mathrm{~m} \times 0.25 \mu \mathrm{m}$ DF of capillary column. Ultra-high purity helium (99.99\%) was used as carrier gas at a constant flow rate of $1.0 \mathrm{ml} / \mathrm{min}$. The injection, transfer line and ion source temperatures were at all $290{ }^{\circ} \mathrm{C}$. The ionizing energy was $70 \mathrm{eV}$. Electron multiplier voltage was obtained from autotune. The oven temperature was programmed from $60^{\circ} \mathrm{C}$ (hold for $2 \mathrm{~min}$ ) to $320^{\circ} \mathrm{C}$ at a rate of $3{ }^{\circ} \mathrm{C} / \mathrm{min}$. The crude sample was diluted with an appropriate solvent $(1 / 10, v / v)$ and filtered. The particle-free diluted crude extracts $(1 \mu \mathrm{L})$ were taken in a syringe and injected into injector with a split ratio 30:1. All data were obtained by collecting the full-scan mass spectra within the scan range 30-600 amu. The percentage composition of the crude extract constituents was expressed as a percentage by peak area. The identification and characterization of chemical compounds in ethanolic crude extract were based on GC retention time. AMDIS and NIST Version-Year 2011 was used MS data library and comparing the spectrum obtained through GC-MS compounds present in the plant's sample was identified.

\section{Glycolate oxidase enzyme inhibition assay}

Glycolate oxidase enzyme inhibition activity was performed in cuvette with a 1-cm light path using a UV-spectrophotometer. Each assay contained $200 \mu \mathrm{M}$ potassium phosphate $(\mathrm{pH} 7.0), 1 \mathrm{mg}$ of bovine serum albumin, $3 \mu \mathrm{M}$ EDTA, $0.1 \mu \mathrm{M}$ DCIP, enzyme and water to a volume of $3 \mathrm{ml}$ and a solution of test plant extracts in DMSO was incubated at room temperature for $15 \mathrm{~min}$. The reaction was started by the addition of $2 \mu \mathrm{M}$ Sodium glycolate was then followed by measure of the decrease in absorbance at $600 \mathrm{~nm}$. The inhibitory activity of each test compound was indicated by their IC50 values calculated using a linear regression curve. The percent inhibition of enzyme activity was calculated using standard formula [22].

$$
\text { Percent of Inhibition (\%) }=\frac{\text { Abs Control }- \text { Abs Sample }}{\text { Abs control }} \times 100
$$

Where control represents reaction mixture as described above excluding test compounds instead contain DMSO only whereas sample represents reaction mixture same as described in method.

\section{Lactate dehydrogenase enzyme inhibition assay}

Lactate dehydrogenase activity was assayed at $\mathrm{pH} 7.4$ by measuring the decrease in absorbance at $340 \mathrm{~nm}$ associated with $\mathrm{NADH}$ oxidation. Assay mixture contained $0.3 \mathrm{mmol} \mathrm{NADH}, 2.0 \mathrm{mmol}$ pyruvate and $100 \mu \mathrm{L}$ enzyme in volume of $3.0 \mathrm{ml}$. The reaction was started by the addition of enzyme. Lactate dehydrogenase inhibitory activity of test plant extracts was monitored spectrophotometrically following the absorbance at $340 \mathrm{~nm}$ under aerobic condition. The reaction mixture containing $0.3 \mathrm{mmol} \mathrm{NADH}, 100 \mu \mathrm{L}$ enzymes in volume of $3.0 \mathrm{ml}$ and a solution of test plant extracts in DMSO was incubated at room temperature for $15 \mathrm{~min}$. The reaction was started by addition of $2 \mathrm{mmol}$ pyruvate and l-lactate formation was then followed by measure of decrease in absorbance at $340 \mathrm{~nm}$. The inhibitory activity of each test compound was indicated by their IC50 values calculated using linear regression curve. The percent inhibition of enzyme activity was calculated using standard formula [22].

$$
\text { Percent of Inhibition }(\%)=\frac{\text { Abs Control }- \text { Abs Sample }}{\text { Abs control }} \times 100
$$

Where control represents reaction mixture as described above excluding test compounds instead contain DMSO only whereas sample represents reaction mixture same as described in method.

\section{ADMET predictions}

ADMET (Absorption, Distribution, Metabolism, Excretion and Toxicity) analyses constitute the pharmacokinetics of a drug molecule [23]. In this study, prediction and significant descriptors of drug-likeness such as mutagenicity, toxicological dosage level and pharmacologically relevant properties of the compounds were predicted using Swissadme (http://www.swissadme.ch) and admetSAR (lmmd.ecust.edu.cn: 8000) servers.

\section{RESULTS AND DISCUSSION}

\section{Flavonoid extraction from Bergenia ciliata (rhizome)}

The ethyl acetate fraction of Bergenia ciliata (rhizome) showed many peaks in the chromatogram (fig. 2a), however, only twelve compounds could be identified and characterized (fig. 2b). Plants contain so many phytoconstituents, many of which are biologically active compounds and are responsible for pharmacological activities [24]. The bioactive secondary metabolites have been shown to reduce the risk and progression of diseases such as cancer, renal disorders, etc., through various biological mechanisms. A total of 12 volatile chemical compounds belonging to hydrocarbons, esters, alcohols, fatty acids, ketones, etc. were identified and characterized in ethyl acetate fraction through GC-MS analysis (table 1).

\section{Glycolate oxidase and lactate dehydrogenase inhibition and mode of inhibition Lineweaver-Burk plots}

The experimental evidence indicates that ethyl acetate fraction of Bergenia ciliata showed a good activity profile for inhibition of glycolate oxidase and lactate dehydrogenase as indicated by IC50 ( $\mu \mathrm{M}$ ) (fig. 3; table 2). To determine the mode of inhibition by active compounds from the plants, Lineweaver-Burk plot analysis was performed [25]. This kinetics study was carried out in the absence and presence of active compounds with varying concentrations of substrate. The initial velocity was expressed as the absorbance decrease at $340 \mathrm{~nm}$ for lactate dehydrogenase and $600 \mathrm{~nm}$ for glycolate oxidase per $10 \mathrm{~s}$ in the assay. In case of glycolate oxidase and lactate dehydrogenase inhibition, B. ciliata (ethyl acetate fraction) were found to be non-competitive inhibitors as Km values were constant while Vmax consequently decreased with increased inhibitor concentration. These finding suggest that inhibition of glycolate oxidase and lactate dehydrogenase activity, leading to retardation of oxalate synthesis, is one of the mechanism through which the plant tested in this study could be exhibiting their antiurolithiac effect. Modulation of glycolate oxidase and lactate dehydrogenase activity by compounds in these extracts would thus eventually lead to a lowering of oxalate content. Presence of glycolate oxidase and lactate dehydrogenase inhibitor blocks the normal pathway of conversion of glyoxylate to oxalate which further form a complex with calcium to lead into the formation of calcium oxalate stones. Thus, oxalate synthesis can be blocked in hyperoxaluria conditions with glycolate oxidase and lactate dehydrogenase inhibitors. This glycolate oxidase and lactate dehydrogenase inhibition could occur in a concentration-dependent or independent manner depending upon the bioactive compounds. Inhibition of glycolate oxidase and lactate dehydrogenase may occur due to these phytoconstituents. This is the first report as per author's knowledge. 


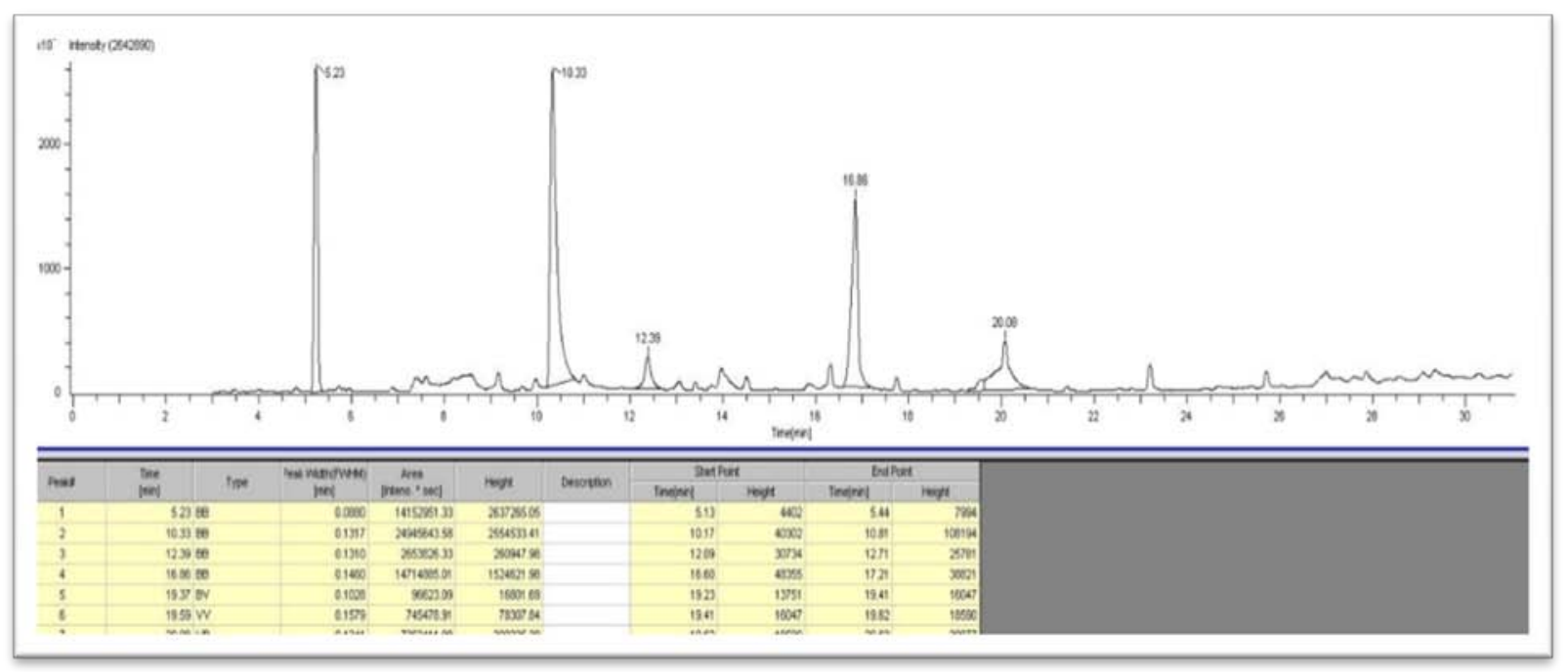

Fig. 2a: Total ion chromatogram (GC-MS) of Bergenia ciliata (rhizome) (ethyl acetate fraction)

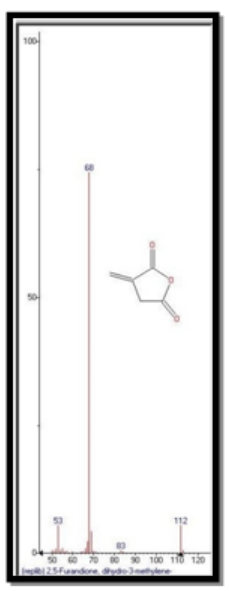

[1]

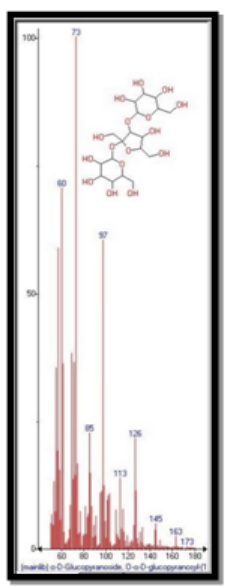

[2]

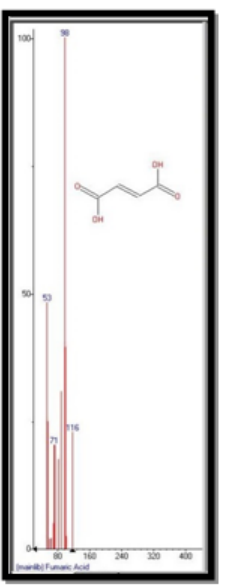

[3]

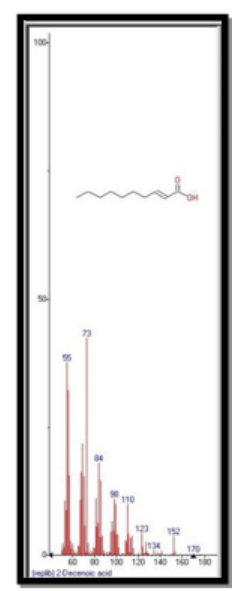

[4]

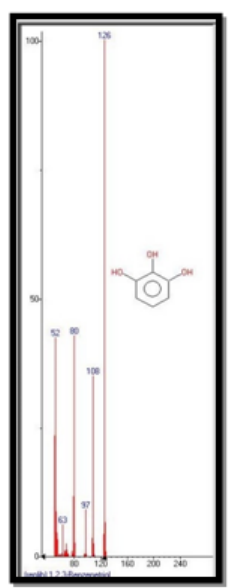

[5]

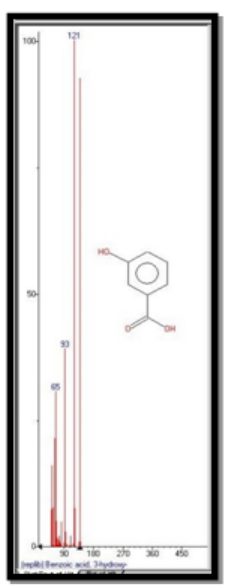

[6]

[1]: 2,5-Furandione, dihydro-3-methylene-[2]: $\alpha$-D-Glucopyranoside,O- $\alpha$-D-glucopyranosyl-(fwdarw 3)- $\beta$-D-fructofuranosyl, [3]: Fumaric acid, [4]: 2-decenoic acid, [5]: 1,2,3-Benzenetriol, [6]: Benzoic acid, 3-hydroxy-

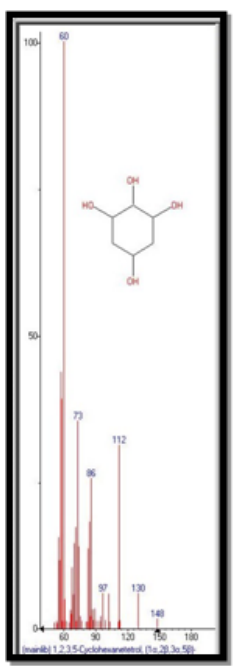

[7]

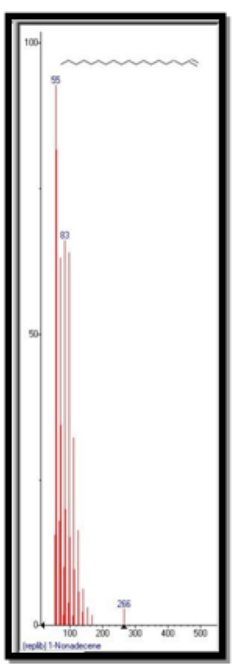

[8]

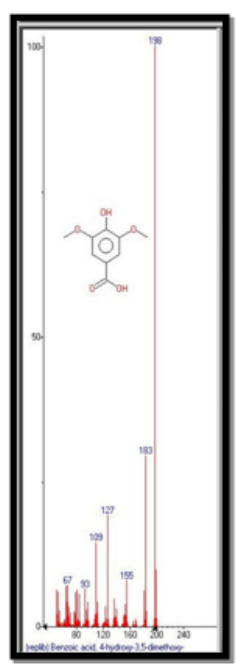

[9]

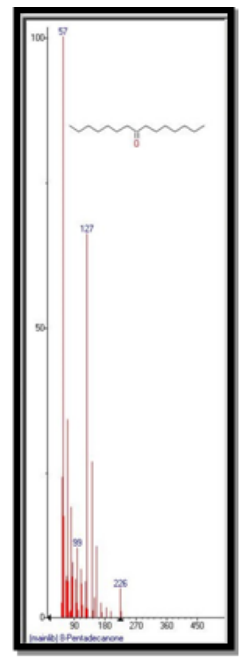

[10]

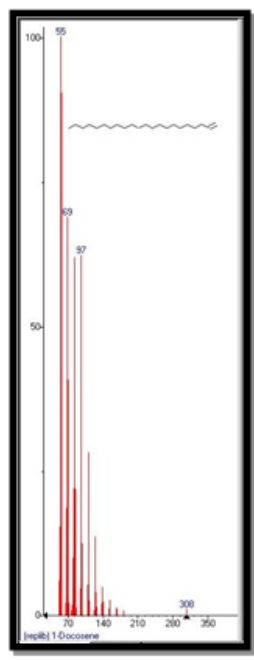

[11]

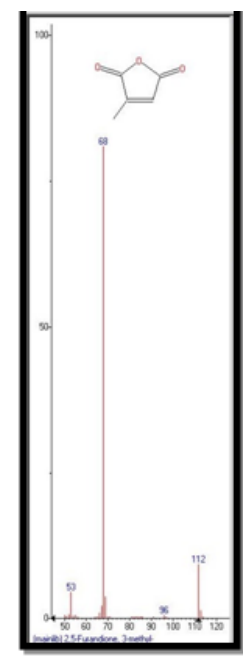

[12]

[7]: 1,2,3,5-Cyclohexanetetrol, $(1 \alpha, 2 \beta, 3 \alpha, 5 \beta)$, [8]: Nonadecane, [9]: Benzoic acid, 4-hydroxy-3,5-dimethoxy-, [10]: 8-pentadecanone, [11]: Docosene, [12]: 2,5- Furandione,3-methyl-

Fig. 2b: Ion chromatograms (GC-MS) of identified compounds from Bergenia ciliata (rhizome) (ethyl acetate fraction) 
Table 1: Bioactivity of phytocomponents identified in the ethyl acetate fraction of Bergenia ciliata

\begin{tabular}{|c|c|}
\hline \multicolumn{2}{|l|}{ Bergenia ciliata (Ethyl acetate fraction) } \\
\hline Name of the compound & Biological Activity** \\
\hline Fumaric acid & $\begin{array}{l}\text { Acidifier, Acidulant, Arachidonic acid-inhibitor, Increase aromatic } \\
\text { amino acid Decarboxylase activity, Inhibit production of uric acid, } \\
\text { Urinary-Acidulant, Urine-acidifier. }\end{array}$ \\
\hline \multirow{3}{*}{$\begin{array}{l}\alpha \text {-D-Glucopyranoside, 0- } \alpha \text {-D-glucopyranosyl-(fwdarw 3)- } \beta \text {-D- } \\
\text { fructofuranosyl }\end{array}$} & Aldehyde-oxidase-inhibitor, Anticancer, Antidote, Antiretinitic, \\
\hline & Antitumor, Catechol-0-Methyl-Transferase-Inhibitor, Increase \\
\hline & $\begin{array}{l}\text { Osteocalcin, Inhibit Production of Tumor Necrosis Factor, Inhibit } \\
\text { production of uric acid, Lower oxalate, Ionic Channel Opener, NADH- } \\
\text { Oxidase Inhibitor, Nitric Oxide Synthase Inhibitor, Occuloirritant, } \\
\text { Occulotensive, Odontolytic }\end{array}$ \\
\hline 2-decenoic acid & $\begin{array}{l}\text { Acidifier, Acidulant, Arachidonic acid-inhibitor, Increased aromatic } \\
\text { amino acid Decarboxylase activity, Inhibit production of uric acid, } \\
\text { Urinary-Acidulant, Urine-acidifier. }\end{array}$ \\
\hline Benzoic acid, 3-hydroxy- & $\begin{array}{l}\text { 17-beta-hydroxysteroid dehydrogenase-inhibitor, Aryl-Hydrocarbon- } \\
\text { Hydroxylase-Inhibitor, Testosterone-Hydroxylase-Inducer, Acidifier, } \\
\text { Acidulant, Arachidonic acid-inhibitor, Increase aromatic amino acid } \\
\text { Decarboxylase activity, Inhibit production of uric acid, Urinary- } \\
\text { Acidulant, Urine-acidifier. }\end{array}$ \\
\hline Benzoic acid, 4-hydroxy-3,5-dimethoxy- & $\begin{array}{l}\text { Acidifier, Acidulant, Arachidonic acid-inhibitor, Increased aromatic } \\
\text { amino acid Decarboxylase activity, Inhibit production of uric acid, } \\
\text { Urinary-Acidulant, Urine-acidifier, 17-beta-hydroxysteroid } \\
\text { dehydrogenase-inhibitor, Aryl-Hydrocarbon-Hydroxylase-Inhibitor, } \\
\text { Testosterone-Hydroxylase-Inducer. }\end{array}$ \\
\hline \multirow[t]{2}{*}{ 2,5-Furandione,3-methyl- } & Catechol-O-Methyl-Transferase-Inhibitor, Catechol-O- \\
\hline & $\begin{array}{l}\text { Methyltransferase-Inhibitor, Methyl-Donar, Methyl-Guanidine- } \\
\text { inhibitor. }\end{array}$ \\
\hline
\end{tabular}

(**Activity source: Dr. Duke's Phytochemical and Ethnobotanical Database)

Table 2: Vmax and Km of B. ciliata (ethyl acetate extracts) for glycolate oxidase (GOX) and lactate dehydrogenase (LDH) inhibition

\begin{tabular}{|c|c|c|c|c|c|}
\hline Samples & Concentrations $(\mu \mathrm{g} / \mathrm{ml})$ & $V_{\max }(\mu \mathrm{g} / \mathrm{min})$ & $K_{m}(\mu g / m l)$ & Type of Inhibition & $\mathrm{IC}_{50}$ value $(\mu \mathrm{g} / \mathrm{ml})$ \\
\hline \multirow[t]{5}{*}{ B. ciliata (gox) } & 10 & 0.025 & 0.86 & Non-competitive & 65.76 \\
\hline & 25 & 0.025 & 0.86 & & \\
\hline & 50 & 0.029 & 0.8 & & \\
\hline & 75 & 0.0196 & 0.8 & & \\
\hline & 100 & 0.0181 & 0.8 & & \\
\hline \multirow[t]{5}{*}{ B. ciliata (ldh) } & 10 & 3.33 & 1.75 & Non-competitive & 69.84 \\
\hline & 25 & 1.66 & 1.75 & & \\
\hline & 50 & 1.25 & 1.63 & & \\
\hline & 75 & 1.25 & 1.63 & & \\
\hline & 100 & 1.42 & 1.63 & & \\
\hline
\end{tabular}

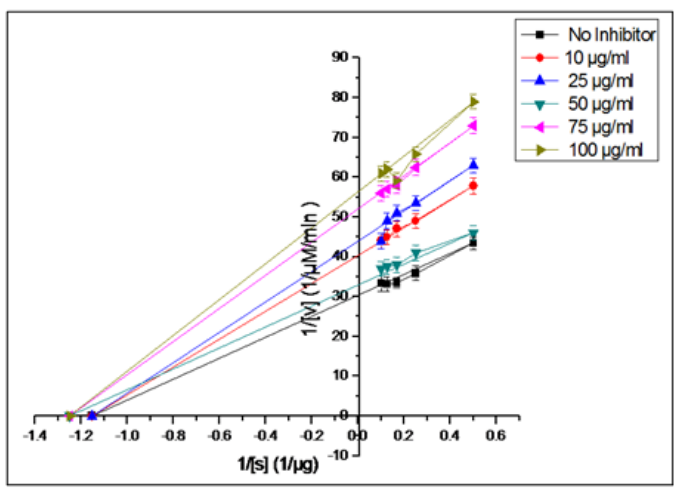

[a] Ethyl acetate fractions of B.ciliata

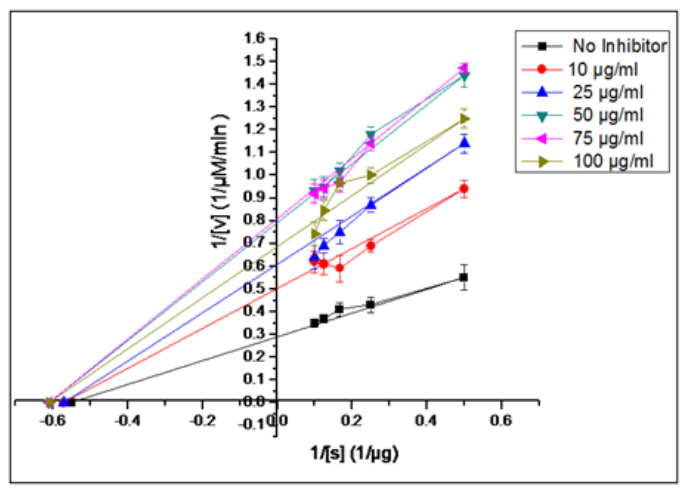

$\lceil\mathrm{b}\rceil$ Ethyl acetate fractions of B.ciliata

Fig. 3: Lineweaver-Burk plot for enzyme Glycolate oxidase and Lactate dehydrogenase

\section{ADMET predictions}

The potential ADME profiles of the compounds as predicted using the admetSAR server, while the distribution profile of the compounds as obtained from the admetSAR server is shown in (table 11). Computational study for the prediction of the relevant properties influencing bioactivity of the lead compounds was performed. The ADME properties of the compounds were evaluated, and the selected properties are linked to metabolism and cell permeation. 
Compound 1: 1,2,3-Benzenetriol

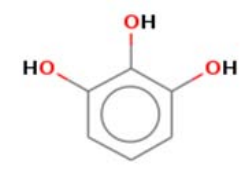

ADMET predicted profile---Classification

\begin{tabular}{|c|c|c|}
\hline Model & Result & Probability \\
\hline \multicolumn{3}{|l|}{ Absorption } \\
\hline Blood-Brain Barrier & BBB- & 0.6478 \\
\hline Human Intestinal Absorption & $\mathrm{HIA}+$ & 0.9642 \\
\hline Caco-2 Permeability & Caco2+ & 0.7355 \\
\hline P-glycoprotein Substrate & Non-substrate & 0.6749 \\
\hline \multirow[t]{2}{*}{ P-glycoprotein Inhibitor } & Non-inhibitor & 0.9691 \\
\hline & Non-inhibitor & 0.9916 \\
\hline Renal Organic Cation Transporter & Non-inhibitor & 0.9289 \\
\hline \multicolumn{3}{|l|}{ Distribution } \\
\hline Subcellular localization & Mitochondria & 0.6807 \\
\hline \multicolumn{3}{|l|}{ Metabolism } \\
\hline CYP450 2C9 Substrate & Non-substrate & 0.8234 \\
\hline CYP450 2D6 Substrate & Non-substrate & 0.9031 \\
\hline CYP450 3A4 Substrate & Non-substrate & 0.7441 \\
\hline CYP450 1A2 Inhibitor & Non-inhibitor & 0.7436 \\
\hline CYP450 2C9 Inhibitor & Non-inhibitor & 0.8271 \\
\hline CYP450 2D6 Inhibitor & Non-inhibitor & 0.9494 \\
\hline CYP450 2C19 Inhibitor & Non-inhibitor & 0.9397 \\
\hline CYP450 3A4 Inhibitor & Non-inhibitor & 0.8682 \\
\hline CYP Inhibitory Promiscuity & Low CYP Inhibitory Promiscuity & 0.6899 \\
\hline \multicolumn{3}{|l|}{ Excretion } \\
\hline \multicolumn{3}{|l|}{ Toxicity } \\
\hline \multirow[t]{2}{*}{ Human Ether-a-go-go-Related Gene Inhibition } & Weak inhibitor & 0.9526 \\
\hline & Non-inhibitor & 0.9299 \\
\hline AMES Toxicity & AMES toxic & 0.7459 \\
\hline Carcinogens & Non-carcinogens & 0.8816 \\
\hline Fish Toxicity & High FHMT & 0.6928 \\
\hline Tetrahymena Pyriformis Toxicity & High TPT & 0.8900 \\
\hline Honey Bee Toxicity & High HBT & 0.6927 \\
\hline Biodegradation & Ready biodegradable & 0.6003 \\
\hline Acute Oral Toxicity & III & 0.8089 \\
\hline Carcinogenicity (Three-class) & Non-required & 0.5339 \\
\hline
\end{tabular}

ADMET predicted profile---Regression

\begin{tabular}{lll}
\hline Model & Value & Unit \\
\hline Absorption & & LogS \\
Aqueous solubility & -0.4107 & LogPapp, cm/s \\
Caco-2 Permeability & 0.5771 & \\
Distribution & & \\
Metabolism & & \\
Excretion & & LD50, mol/kg \\
Toxicity & & pLC50, mg/l \\
Rat Acute Toxicity & 2.2344 & pIGC50, ug/l \\
Fish Toxicity & 1.1417 & 0.5649 \\
Tetrahymena Pyriformis Toxicity & & \\
\hline
\end{tabular}

Compound 2: 2-decenoic acid

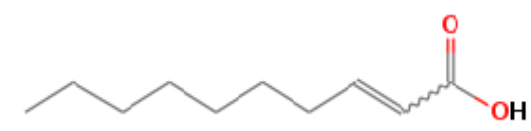

ADMET predicted profile---Classification

\begin{tabular}{lll}
\hline Model & Result & Probability \\
\hline Absorption & & \\
Blood-Brain Barrier & BBB+ & 0.9638 \\
Human Intestinal Absorption & HIA+ & 0.9908 \\
Caco-2 Permeability & Caco2+ & 0.8326 \\
P-glycoprotein Substrate & Non-substrate & 0.6523 \\
P-glycoprotein Inhibitor & Non-inhibitor & 0.9689 \\
& Non-inhibitor & 0.7699 \\
\hline
\end{tabular}




\begin{tabular}{|c|c|c|}
\hline Model & Result & Probability \\
\hline Renal Organic Cation Transporter & Non-inhibitor & 0.9061 \\
\hline \multicolumn{3}{|l|}{ Distribution } \\
\hline Subcellular localization & Plasma membrane & 0.6894 \\
\hline \multicolumn{3}{|l|}{ Metabolism } \\
\hline CYP450 2C9 Substrate & Non-substrate & 0.7322 \\
\hline CYP450 2D6 Substrate & Non-substrate & 0.9059 \\
\hline CYP450 3A4 Substrate & Non-substrate & 0.7043 \\
\hline CYP450 1A2 Inhibitor & Inhibitor & 0.7152 \\
\hline CYP450 2C9 Inhibitor & Non-inhibitor & 0.8948 \\
\hline CYP450 2D6 Inhibitor & Non-inhibitor & 0.9474 \\
\hline CYP450 2C19 Inhibitor & Non-inhibitor & 0.9459 \\
\hline CYP450 3A4 Inhibitor & Non-inhibitor & 0.9608 \\
\hline CYP Inhibitory Promiscuity & Low CYP Inhibitory Promiscuity & 0.8882 \\
\hline \multicolumn{3}{|l|}{ Excretion } \\
\hline \multicolumn{3}{|l|}{ Toxicity } \\
\hline \multirow[t]{2}{*}{ Human Ether-a-go-go-Related Gene Inhibition } & Weak inhibitor & 0.8983 \\
\hline & Non-inhibitor & 0.9369 \\
\hline AMES Toxicity & Non AMES toxic & 0.9737 \\
\hline Carcinogens & Non-carcinogens & 0.5263 \\
\hline Fish Toxicity & High FHMT & 0.9784 \\
\hline Tetrahymena Pyriformis Toxicity & High TPT & 0.9996 \\
\hline Honey Bee Toxicity & High HBT & 0.7359 \\
\hline Biodegradation & Ready biodegradable & 0.7618 \\
\hline Acute Oral Toxicity & III & 0.8593 \\
\hline Carcinogenicity (Three-class) & Non-required & 0.6623 \\
\hline
\end{tabular}

Carcinogenicity (Three-class)

ADMET predicted profile---Regression

\begin{tabular}{lll}
\hline Model & Value & Unit \\
\hline Absorption & & LogS \\
Aqueous solubility & -3.5855 & LogPapp, cm/s \\
Caco-2 Permeability & 1.3217 & \\
Distribution & & \\
Metabolism & & \\
Excretion & & LD50, mol/kg \\
Toxicity & & pLC50, mg/l \\
Rat Acute Toxicity & 1.9685 & pIGC50, ug/l \\
Fish Toxicity & 0.8959 & 0.7180 \\
Tetrahymena Pyriformis Toxicity & & \\
\hline
\end{tabular}

Compound 3: 8-pentadecanone

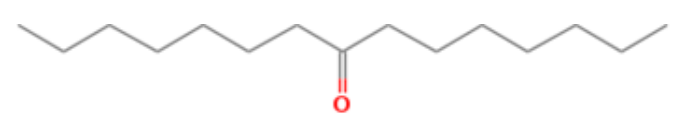

ADMET predicted profile---Classification

\begin{tabular}{|c|c|c|}
\hline Model & Result & Probability \\
\hline \multicolumn{3}{|l|}{ Absorption } \\
\hline Blood-Brain Barrier & $\mathrm{BBB}+$ & 0.9882 \\
\hline Human Intestinal Absorption & HIA+ & 0.9955 \\
\hline Caco-2 Permeability & Caco2+ & 0.8766 \\
\hline P-glycoprotein Substrate & Non-substrate & 0.6680 \\
\hline \multirow[t]{2}{*}{ P-glycoprotein Inhibitor } & Non-inhibitor & 0.8320 \\
\hline & Non-inhibitor & 0.7768 \\
\hline Renal Organic Cation Transporter & Non-inhibitor & 0.8727 \\
\hline \multicolumn{3}{|l|}{ Distribution } \\
\hline Subcellular localization & Mitochondria & 0.4585 \\
\hline \multicolumn{3}{|l|}{ Metabolism } \\
\hline CYP450 2C9 Substrate & Non-substrate & 0.8589 \\
\hline CYP450 2D6 Substrate & Non-substrate & 0.8439 \\
\hline CYP450 3A4 Substrate & Non-substrate & 0.6531 \\
\hline CYP450 1A2 Inhibitor & Inhibitor & 0.6890 \\
\hline CYP450 2C9 Inhibitor & Non-inhibitor & 0.9433 \\
\hline CYP450 2D6 Inhibitor & Non-inhibitor & 0.9502 \\
\hline CYP450 2C19 Inhibitor & Non-inhibitor & 0.9645 \\
\hline CYP450 3A4 Inhibitor & Non-inhibitor & 0.9815 \\
\hline CYP Inhibitory Promiscuity & Low CYP Inhibitory Promiscuity & 0.8752 \\
\hline \multicolumn{3}{|l|}{ Excretion } \\
\hline \multicolumn{3}{|l|}{ Toxicity } \\
\hline \multirow[t]{2}{*}{ Human Ether-a-go-go-Related Gene Inhibition } & Weak inhibitor & 0.8043 \\
\hline & Non-inhibitor & 0.7659 \\
\hline
\end{tabular}




\begin{tabular}{|c|c|c|c|}
\hline Model & \multicolumn{2}{|c|}{ Result } & Probability \\
\hline AMES Toxicity & \multicolumn{2}{|c|}{ Non AMES toxic } & 0.9859 \\
\hline Carcinogens & \multicolumn{2}{|c|}{ Carcinogens } & 0.6310 \\
\hline Fish Toxicity & \multicolumn{2}{|c|}{ High FHMT } & 0.7423 \\
\hline Tetrahymena Pyriformis Toxicity & \multicolumn{2}{|c|}{ High TPT } & 0.8910 \\
\hline Honey Bee Toxicity & \multicolumn{2}{|c|}{ High HBT } & 0.7254 \\
\hline Biodegradation & \multicolumn{2}{|c|}{ Ready biodegradable } & 0.8731 \\
\hline Acute Oral Toxicity & \multicolumn{2}{|c|}{ III } & 0.8455 \\
\hline Carcinogenicity (Three-class) & \multicolumn{2}{|c|}{ Non-required } & 0.7622 \\
\hline \multicolumn{4}{|c|}{ ADMET predicted profile---Regression } \\
\hline Model & Value & Unit & \\
\hline \multicolumn{4}{|l|}{ Absorption } \\
\hline Aqueous solubility & -2.2537 & $\log S$ & \\
\hline Caco-2 Permeability & 1.3709 & LogPapp, $\mathrm{cm} / \mathrm{s}$ & \\
\hline \multicolumn{4}{|l|}{ Distribution } \\
\hline \multicolumn{4}{|l|}{ Metabolism } \\
\hline \multicolumn{4}{|l|}{ Excretion } \\
\hline \multicolumn{4}{|l|}{ Toxicity } \\
\hline Rat Acute Toxicity & 1.5870 & LD50, mol/kg & \\
\hline Fish Toxicity & 0.8094 & $\mathrm{pLC} 50, \mathrm{mg} / \mathrm{l}$ & \\
\hline Tetrahymena Pyriformis Toxicity & 0.5873 & pIGC50, ug/l & \\
\hline
\end{tabular}

Compound 4: Benzoic acid, 3-hydroxy-

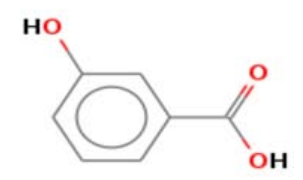

ADMET predicted profile---Classification

\begin{tabular}{|c|c|c|}
\hline Model & Result & Probability \\
\hline \multicolumn{3}{|l|}{ Absorption } \\
\hline Blood-Brain Barrier & $\mathrm{BBB}+$ & 0.5320 \\
\hline Human Intestinal Absorption & HIA+ & 0.9872 \\
\hline Caco-2 Permeability & Caco2+ & 0.8937 \\
\hline P-glycoprotein Substrate & Non-substrate & 0.7493 \\
\hline \multirow{2}{*}{ P-glycoprotein Inhibitor } & Non-inhibitor & 0.9890 \\
\hline & Non-inhibitor & 0.9927 \\
\hline Renal Organic Cation Transporter & Non-inhibitor & 0.9078 \\
\hline \multicolumn{3}{|l|}{ Distribution } \\
\hline Subcellular localization & Mitochondria & 0.9063 \\
\hline \multicolumn{3}{|l|}{ Metabolism } \\
\hline CYP450 2C9 Substrate & Non-substrate & 0.8115 \\
\hline CYP450 2D6 Substrate & Non-substrate & 0.9377 \\
\hline CYP450 3A4 Substrate & Non-substrate & 0.7652 \\
\hline CYP450 1A2 Inhibitor & Non-inhibitor & 0.9752 \\
\hline CYP450 2C9 Inhibitor & Non-inhibitor & 0.9697 \\
\hline CYP450 2D6 Inhibitor & Non-inhibitor & 0.9827 \\
\hline CYP450 2C19 Inhibitor & Non-inhibitor & 0.9651 \\
\hline CYP450 3A4 Inhibitor & Non-inhibitor & 0.9493 \\
\hline CYP Inhibitory Promiscuity & Low CYP Inhibitory Promiscuity & 0.9554 \\
\hline \multicolumn{3}{|l|}{ Excretion } \\
\hline \multicolumn{3}{|l|}{ Toxicity } \\
\hline \multirow[t]{2}{*}{ Human Ether-a-go-go-Related Gene Inhibition } & Weak inhibitor & 0.9617 \\
\hline & Non-inhibitor & 0.9771 \\
\hline AMES Toxicity & Non AMES toxic & 0.9826 \\
\hline Carcinogens & Non-carcinogens & 0.8226 \\
\hline Fish Toxicity & High FHMT & 0.7616 \\
\hline Tetrahymena Pyriformis Toxicity & Low TPT & 0.8365 \\
\hline Honey Bee Toxicity & High HBT & 0.7797 \\
\hline Biodegradation & Ready biodegradable & 0.8413 \\
\hline Acute Oral Toxicity & III & 0.5472 \\
\hline Carcinogenicity (Three-class) & Non-required & 0.6300 \\
\hline
\end{tabular}

ADMET predicted profile---Regression

\begin{tabular}{lll}
\hline Model & Value & Unit \\
\hline Absorption & & \\
Aqueous solubility & -1.3479 & $\operatorname{LogS}$ \\
Caco-2 Permeability & 1.1511 & $\operatorname{LogPapp}, \mathrm{cm} / \mathrm{s}$ \\
\hline
\end{tabular}




\begin{tabular}{lll}
\hline Model & Value & Unit \\
\hline Distribution & & \\
Metabolism & & \\
Excretion & & \\
Toxicity & & LD50, mol/kg \\
Rat Acute Toxicity & 1.3983 & pLC50, mg/l \\
Fish Toxicity & 2.2036 & pIGC50,ug/l \\
Tetrahymena Pyriformis Toxicity & -0.8949 & \\
\hline
\end{tabular}

\section{Compound 5: Benzoic acid, 4-hydroxy-3,5-dimethoxy}<smiles>COc1cc(C(=O)O)cc(OC)c1O</smiles>

ADMET predicted profile---Classification

\begin{tabular}{|c|c|c|}
\hline Model & Result & Probability \\
\hline \multicolumn{3}{|l|}{ Absorption } \\
\hline Blood-Brain Barrier & $\mathrm{BBB}+$ & 0.5861 \\
\hline Human Intestinal Absorption & HIA+ & 0.9165 \\
\hline Caco-2 Permeability & Caco $2+$ & 0.7124 \\
\hline P-glycoprotein Substrate & Non-substrate & 0.6033 \\
\hline \multirow{2}{*}{ P-glycoprotein Inhibitor } & Non-inhibitor & 0.9199 \\
\hline & Non-inhibitor & 0.8879 \\
\hline Renal Organic Cation Transporter & Non-inhibitor & 0.9136 \\
\hline \multicolumn{3}{|l|}{ Distribution } \\
\hline Subcellular localization & Mitochondria & 0.8825 \\
\hline \multicolumn{3}{|l|}{ Metabolism } \\
\hline CYP450 2C9 Substrate & Non-substrate & 0.8213 \\
\hline CYP450 2D6 Substrate & Non-substrate & 0.8899 \\
\hline CYP450 3A4 Substrate & Non-substrate & 0.6258 \\
\hline CYP450 1A2 Inhibitor & Non-inhibitor & 0.9052 \\
\hline CYP450 2C9 Inhibitor & Non-inhibitor & 0.9316 \\
\hline CYP450 2D6 Inhibitor & Non-inhibitor & 0.9445 \\
\hline CYP450 2C19 Inhibitor & Non-inhibitor & 0.8579 \\
\hline CYP450 3A4 Inhibitor & Non-inhibitor & 0.9538 \\
\hline CYP Inhibitory Promiscuity & Low CYP Inhibitory Promiscuity & 0.8767 \\
\hline \multicolumn{3}{|l|}{ Excretion } \\
\hline \multicolumn{3}{|l|}{ Toxicity } \\
\hline \multirow[t]{2}{*}{ Human Ether-a-go-go-Related Gene Inhibition } & Weak inhibitor & 0.9858 \\
\hline & Non-inhibitor & 0.9664 \\
\hline AMES Toxicity & Non AMES toxic & 0.9342 \\
\hline Carcinogens & Non-carcinogens & 0.8809 \\
\hline Fish Toxicity & High FHMT & 0.8272 \\
\hline Tetrahymena Pyriformis Toxicity & High TPT & 0.9067 \\
\hline Honey Bee Toxicity & High HBT & 0.7522 \\
\hline Biodegradation & Ready biodegradable & 0.7199 \\
\hline Acute Oral Toxicity & II & 0.4765 \\
\hline Carcinogenicity (Three-class) & Non-required & 0.7159 \\
\hline
\end{tabular}

ADMET predicted profile---Regression

\begin{tabular}{lll}
\hline Model & Value & Unit \\
\hline Absorption & & LogS \\
Aqueous solubility & -2.1167 & LogPapp, cm/s \\
Caco-2 Permeability & 0.7627 & \\
Distribution & & \\
Metabolism & & \\
Excretion & & LD50, mol/kg \\
Toxicity & & pLC50, mg/l \\
Rat Acute Toxicity & 2.5353 & pIGC50, ug/l \\
Fish Toxicity & 1.6288 & \\
Tetrahymena Pyriformis Toxicity & 0.4613 & \\
\hline
\end{tabular}




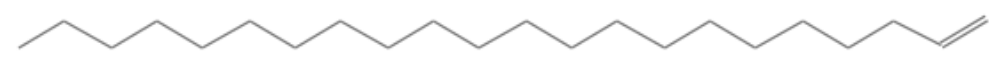

ADMET predicted profile---Classification

\begin{tabular}{|c|c|c|}
\hline Model & Result & Probability \\
\hline \multicolumn{3}{|l|}{ Absorption } \\
\hline Blood-Brain Barrier & $\mathrm{BBB}+$ & 0.9763 \\
\hline Human Intestinal Absorption & HIA+ & 0.9928 \\
\hline Caco-2 Permeability & Caco2+ & 0.7989 \\
\hline P-glycoprotein Substrate & Non-substrate & 0.6522 \\
\hline \multirow{2}{*}{ P-glycoprotein Inhibitor } & Non-inhibitor & 0.7775 \\
\hline & Non-inhibitor & 0.5881 \\
\hline Renal Organic Cation Transporter & Non-inhibitor & 0.8619 \\
\hline \multicolumn{3}{|l|}{ Distribution } \\
\hline Subcellular localization & Lysosome & 0.4578 \\
\hline \multicolumn{3}{|l|}{ Metabolism } \\
\hline CYP450 2C9 Substrate & Non-substrate & 0.8261 \\
\hline CYP450 2D6 Substrate & Non-substrate & 0.8048 \\
\hline CYP450 3A4 Substrate & Non-substrate & 0.7278 \\
\hline CYP450 1A2 Inhibitor & Inhibitor & 0.5418 \\
\hline CYP450 2C9 Inhibitor & Non-inhibitor & 0.9157 \\
\hline CYP450 2D6 Inhibitor & Non-inhibitor & 0.9426 \\
\hline CYP450 2C19 Inhibitor & Non-inhibitor & 0.9190 \\
\hline CYP450 3A4 Inhibitor & Non-inhibitor & 0.9832 \\
\hline CYP Inhibitory Promiscuity & Low CYP Inhibitory Promiscuity & 0.6838 \\
\hline \multicolumn{3}{|l|}{ Excretion } \\
\hline \multicolumn{3}{|l|}{ Toxicity } \\
\hline \multirow[t]{2}{*}{ Human Ether-a-go-go-Related Gene Inhibition } & Weak inhibitor & 0.7812 \\
\hline & Non-inhibitor & 0.8777 \\
\hline AMES Toxicity & Non AMES toxic & 0.9904 \\
\hline Carcinogens & Carcinogens & 0.6000 \\
\hline Fish Toxicity & High FHMT & 0.9954 \\
\hline Tetrahymena Pyriformis Toxicity & High TPT & 0.9981 \\
\hline Honey Bee Toxicity & High HBT & 0.7751 \\
\hline Biodegradation & Ready biodegradable & 0.5000 \\
\hline Acute Oral Toxicity & III & 0.6572 \\
\hline Carcinogenicity (Three-class) & Non-required & 0.5494 \\
\hline
\end{tabular}

ADMET predicted profile---Regression

\begin{tabular}{|c|c|c|}
\hline Model & Value & Unit \\
\hline \multicolumn{3}{|l|}{ Absorption } \\
\hline Aqueous solubility & -4.9876 & $\log S$ \\
\hline Caco-2 Permeability & 1.3776 & LogPapp, $\mathrm{cm} / \mathrm{s}$ \\
\hline \multicolumn{3}{|l|}{ Distribution } \\
\hline \multicolumn{3}{|l|}{ Metabolism } \\
\hline \multicolumn{3}{|l|}{ Excretion } \\
\hline \multicolumn{3}{|l|}{ Toxicity } \\
\hline Rat Acute Toxicity & 1.3452 & LD50, mol/kg \\
\hline Fish Toxicity & -1.1807 & $\mathrm{pLC} 50, \mathrm{mg} / \mathrm{l}$ \\
\hline Tetrahymena Pyriformis Toxicity & 1.4804 & pIGC50, ug/l \\
\hline
\end{tabular}

\section{Compound 7: fumaric acid}

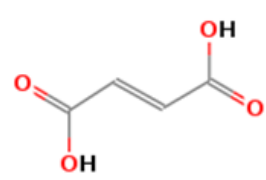

ADMET predicted profile---Classification

\begin{tabular}{lll}
\hline Model & Result & Probability \\
\hline Absorption & & \\
Blood-Brain Barrier & BBB+ & 0.9017 \\
Human Intestinal Absorption & HIA+ & 0.8740 \\
Caco-2 Permeability & Caco2- & 0.6728 \\
P-glycoprotein Substrate & Non-substrate & 0.8006 \\
P-glycoprotein Inhibitor & Non-inhibitor & 0.9850 \\
Renal Organic Cation Transporter & Non-inhibitor & 0.9808 \\
Distribution & Non-inhibitor & 0.9583 \\
\hline
\end{tabular}




\begin{tabular}{|c|c|c|}
\hline Model & Result & Probability \\
\hline Subcellular localization & Mitochondria & 0.7863 \\
\hline \multicolumn{3}{|l|}{ Metabolism } \\
\hline CYP450 2C9 Substrate & Non-substrate & 0.8262 \\
\hline CYP450 2D6 Substrate & Non-substrate & 0.9397 \\
\hline CYP450 3A4 Substrate & Non-substrate & 0.8039 \\
\hline CYP450 1A2 Inhibitor & Non-inhibitor & 0.9659 \\
\hline CYP450 2C9 Inhibitor & Non-inhibitor & 0.9490 \\
\hline CYP450 2D6 Inhibitor & Non-inhibitor & 0.9606 \\
\hline CYP450 2C19 Inhibitor & Non-inhibitor & 0.9773 \\
\hline CYP450 3A4 Inhibitor & Non-inhibitor & 0.9554 \\
\hline CYP Inhibitory Promiscuity & Low CYP Inhibitory Promiscuity & 0.9899 \\
\hline \multicolumn{3}{|l|}{ Excretion } \\
\hline \multicolumn{3}{|l|}{ Toxicity } \\
\hline \multirow[t]{2}{*}{ Human Ether-a-go-go-Related Gene Inhibition } & Weak inhibitor & 0.9836 \\
\hline & Non-inhibitor & 0.9891 \\
\hline AMES Toxicity & Non AMES toxic & 0.9132 \\
\hline Carcinogens & Non-carcinogens & 0.5130 \\
\hline Fish Toxicity & High FHMT & 0.8398 \\
\hline Tetrahymena Pyriformis Toxicity & Low TPT & 0.9808 \\
\hline Honey Bee Toxicity & High HBT & 0.7308 \\
\hline Biodegradation & Ready biodegradable & 0.7561 \\
\hline Acute Oral Toxicity & III & 0.7762 \\
\hline Carcinogenicity (Three-class) & Non-required & 0.7191 \\
\hline
\end{tabular}

ADMET predicted profile---Regression

\begin{tabular}{lll}
\hline Model & Value & Unit \\
\hline Absorption & & LogS \\
Aqueous solubility & -0.3321 & LogPapp, cm/s \\
Caco-2 Permeability & 0.4098 & \\
Distribution & & \\
Metabolism & & \\
Excretion & & LD50, mol/kg \\
Toxicity & & pLC50, mg/l \\
Rat Acute Toxicity & 1.6871 & pIGC50, ug/l \\
Fish Toxicity & 0.9694 & -0.6339 \\
Tetrahymena Pyriformis Toxicity & & \\
\hline
\end{tabular}

\section{Compound 8: Nonadecane}

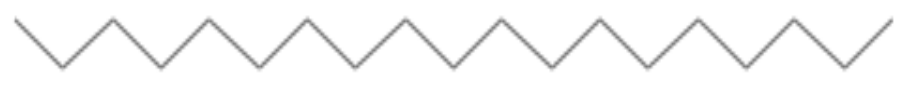

ADMET predicted profile---Classification

\begin{tabular}{|c|c|c|}
\hline Model & Result & Probability \\
\hline \multicolumn{3}{|l|}{ Absorption } \\
\hline Blood-Brain Barrier & $\mathrm{BBB}+$ & 0.9821 \\
\hline Human Intestinal Absorption & $\mathrm{HIA}+$ & 0.9921 \\
\hline Caco-2 Permeability & Caco $2+$ & 0.8284 \\
\hline P-glycoprotein Substrate & Non-substrate & 0.6915 \\
\hline \multirow[t]{2}{*}{ P-glycoprotein Inhibitor } & Non-inhibitor & 0.8985 \\
\hline & Non-inhibitor & 0.7267 \\
\hline Renal Organic Cation Transporter & Non-inhibitor & 0.8780 \\
\hline \multicolumn{3}{|l|}{ Distribution } \\
\hline Subcellular localization & Lysosome & 0.5981 \\
\hline \multicolumn{3}{|l|}{ Metabolism } \\
\hline CYP450 2C9 Substrate & Non-substrate & 0.8480 \\
\hline CYP450 2D6 Substrate & Non-substrate & 0.7762 \\
\hline CYP450 3A4 Substrate & Non-substrate & 0.7237 \\
\hline CYP450 1A2 Inhibitor & Non-inhibitor & 0.6175 \\
\hline CYP450 2C9 Inhibitor & Non-inhibitor & 0.9349 \\
\hline CYP450 2D6 Inhibitor & Non-inhibitor & 0.9373 \\
\hline CYP450 2C19 Inhibitor & Non-inhibitor & 0.9540 \\
\hline CYP450 3A4 Inhibitor & Non-inhibitor & 0.9877 \\
\hline CYP Inhibitory Promiscuity & Low CYP Inhibitory Promiscuity & 0.8149 \\
\hline \multicolumn{3}{|l|}{ Excretion } \\
\hline \multicolumn{3}{|l|}{ Toxicity } \\
\hline \multirow[t]{2}{*}{ Human Ether-a-go-go-Related Gene Inhibition } & Weak inhibitor & 0.8620 \\
\hline & Non-inhibitor & 0.8109 \\
\hline AMES Toxicity & Non AMES toxic & 0.9965 \\
\hline Carcinogens & Carcinogens & 0.6420 \\
\hline
\end{tabular}




\begin{tabular}{lll}
\hline Model & Result & Probability \\
\hline Fish Toxicity & High FHMT & 0.9374 \\
Tetrahymena Pyriformis Toxicity & High TPT & 0.9947 \\
Honey Bee Toxicity & High HBT & 0.7485 \\
Biodegradation & Ready biodegradable & 0.7561 \\
Acute Oral Toxicity & III & 0.6143 \\
Carcinogenicity (Three-class) & Non-required & 0.6328 \\
\hline
\end{tabular}

ADMET predicted profile---Regression

\begin{tabular}{lll}
\hline Model & Value & Unit \\
\hline Absorption & & LogS \\
Aqueous solubility & -5.1776 & LogPapp, cm/s \\
Caco-2 Permeability & 1.3807 & \\
Distribution & & \\
Metabolism & & \\
Excretion & & LD50, mol/kg \\
Toxicity & & pLC50, mg/l \\
Rat Acute Toxicity & 1.3444 & pIGC50, ug/l \\
Fish Toxicity & -0.7109 & \\
Tetrahymena Pyriformis Toxicity & 0.3450 & \\
\hline
\end{tabular}

Compound 9: 2,5-Furandione, dihydro-3-methylene<smiles>C=C1CC(=O)OC1=O</smiles>

ADMET predicted profile---Classification

\begin{tabular}{|c|c|c|}
\hline Model & Result & Probability \\
\hline \multicolumn{3}{|l|}{ Absorption } \\
\hline Blood-Brain Barrier & $\mathrm{BBB}+$ & 0.9627 \\
\hline Human Intestinal Absorption & $\mathrm{HIA}+$ & 0.9833 \\
\hline Caco-2 Permeability & Caco2+ & 0.5452 \\
\hline P-glycoprotein Substrate & Non-substrate & 0.7919 \\
\hline \multirow[t]{2}{*}{ P-glycoprotein Inhibitor } & Non-inhibitor & 0.6266 \\
\hline & Non-inhibitor & 0.9951 \\
\hline Renal Organic Cation Transporter & Non-inhibitor & 0.8772 \\
\hline \multicolumn{3}{|l|}{ Distribution } \\
\hline Subcellular localization & Mitochondria & 0.6548 \\
\hline \multicolumn{3}{|l|}{ Metabolism } \\
\hline CYP450 2C9 Substrate & Non-substrate & 0.8815 \\
\hline CYP450 2D6 Substrate & Non-substrate & 0.8935 \\
\hline CYP450 3A4 Substrate & Non-substrate & 0.7178 \\
\hline CYP450 1A2 Inhibitor & Non-inhibitor & 0.8434 \\
\hline CYP450 2C9 Inhibitor & Non-inhibitor & 0.9264 \\
\hline CYP450 2D6 Inhibitor & Non-inhibitor & 0.9524 \\
\hline CYP450 2C19 Inhibitor & Non-inhibitor & 0.8061 \\
\hline CYP450 3A4 Inhibitor & Non-inhibitor & 0.9487 \\
\hline CYP Inhibitory Promiscuity & Low CYP Inhibitory Promiscuity & 0.9099 \\
\hline \multicolumn{3}{|l|}{ Excretion } \\
\hline \multicolumn{3}{|l|}{ Toxicity } \\
\hline \multirow[t]{2}{*}{ Human Ether-a-go-go-Related Gene Inhibition } & Weak inhibitor & 0.9172 \\
\hline & Non-inhibitor & 0.9875 \\
\hline AMES Toxicity & Non AMES toxic & 0.8025 \\
\hline Carcinogens & Non-carcinogens & 0.8480 \\
\hline Fish Toxicity & High FHMT & 0.9233 \\
\hline Tetrahymena Pyriformis Toxicity & Low TPT & 0.7182 \\
\hline Honey Bee Toxicity & High HBT & 0.8293 \\
\hline Biodegradation & Ready biodegradable & 0.7452 \\
\hline Acute Oral Toxicity & III & 0.7565 \\
\hline Carcinogenicity (Three-class) & Non-required & 0.5918 \\
\hline
\end{tabular}

ADMET predicted profile---Regression

\begin{tabular}{lll}
\hline Model & Value & Unit \\
\hline Absorption & & LogS \\
Aqueous solubility & -0.6464 & LogPapp, cm/s \\
Caco-2 Permeability & 0.9549 & \\
Distribution & & \\
Metabolism & & \\
\hline
\end{tabular}




\begin{tabular}{lll}
\hline Model & Value & Unit \\
\hline Excretion & & \\
Toxicity & & LD50, mol/kg \\
Rat Acute Toxicity & 1.9632 & pLC50, mg/l \\
Fish Toxicity & -0.0057 & pIGC50, ug/l \\
Tetrahymena Pyriformis Toxicity & -0.4252 & \\
\hline
\end{tabular}

Previous study showed that phytochemical constituents of $B$. ciliata are gallic acid, bergenin, (+)-afzelechin [26], 11-0-galloyl bergenin [27], paashaanolactone [26], $\beta$-Sitosterol [28] and $\beta$-Sitosterol- $D$ glucoside [29]. Pharmacological properties have demonstrated that the root is well known in traditional medicine for protection against diarrhea; cough, in uric acid diathesis and in pulmonary infections [28]; coughs and colds, hemorrhoids, asthma and urinary problems [30]. The juice of $B$. ciliata leaves is used as drops to relieve earaches [30]. Currently, Various Ayurvedic classical drugs such as Pashanabhedadi kwath, Pashanabhedadi ghrit, Pashanabhedadi Churan etc. are prepared from Pashanbhed rhizome.

\section{CONCLUSION}

We have extracted, purified flavonoids by the liquid-liquid extraction method. Finally, ethyl acetate fractions were collected and characterized by GC-MS analysis. These fractions are used to perform glycolate oxidase and lactate dehydrogenase inhibition study. Flavonoids fraction of $B$. ciliata showed good enzyme inhibitory activity and ADME profile. Lineviewer-Burk plot and mode of inhibition confirmed the potential of it's as glycolate oxidase and lactate dehydrogenase inhibitors. However, further dose adjustment and molecular mechanism study need to perform for better understanding.

\section{ACKNOWLEDGEMENT}

We would like to offer special thanks to Professor C N Khobragade, who although no longer with us, continue to inspire by his example and dedication to students he served over the course of career.

\section{FUNDING}

Nil

\section{AUTHORS CONTRIBUTIONS}

All the authors have contributed equally.

\section{CONFLICT OF INTERESTS}

Declared none

\section{REFERENCES}

1. Malgorzata E, Anna M, Anna W, Iwona M. Dietary antioxidant and flavonoid intakes are reduced in the elderly. Oxid Med Cell Longev. 2015:Article ID 843173.

2. Li Y, But PP, Ooi VE. Antiviral activity and mode of action of caffeoylquinic acids from Schefflera heptaphylla (L.) frodin. Antiviral Res. 2005;68(1):1-9. doi: 10.1016/j.antiviral.2005.06.004, PMID 16140400.

3. Sousa A, Ferreira IC, Calhelha R, Andrade PB, Valentao P, Seabra R, Estevinho L, Bento A, Pereira JA. Phenolics and antimicrobial activity of traditional stoned table olives 'alcaparra'. Bioorg Med Chem. 2006;14(24):8533-8. doi: 10.1016/j.bmc.2006.08.027, PMID 16971127.

4. Kim KH, Kim YH, Lee KR. Isolation of quinic acid derivatives and flavonoids from the aerial parts of Lactuca indica L. and their hepatoprotective activity in vitro. Bioorg Med Chem Lett. 2007;17(24):6739-43. doi: 10.1016/j.bmcl.2007.10.046, PMID 18029179.

5. Oh H, Kim DH, Cho JH, Kim YC. Hepatoprotective and free radical scavenging activities of phenolic petrosins and flavonoids isolated from Equisetum arvense. J Ethnopharmacol. 2004;95(2-3):421-4. doi: 10.1016/j.jep.2004.08.015, PMID 15507369.

6. Innocenti G, Vegeto E, Dall'Acqua S, Ciana P, Giorgetti M, Agradi E, Sozzi A, Fico G, Tomè F. In vitro estrogenic activity of Achillea millefolium L. Phytomedicine. 2007;14(2-3):147-52. doi: 10.1016/j.phymed.2006.05.005, PMID 16860978.

7. Hamauzu Y, Irie M, Kondo M, Fujita T. Antiulcerative properties of crude polyphenols and juice of apple, and Chinese quince extracts. Food Chem. 2008;108(2):488-95. doi: 10.1016/j.foodchem.2007.10.084, PMID 26059126.

8. Ha CL, Weng CY, Wang L, Lian TW, Wu MJ. Immunomodulatory effect of Glossogyne tenuifolia in murine peritoneal macrophages and splenocytes. J Ethnopharmacol. 2006;107(1):116-25. doi: 10.1016/j.jep.2006.02.015, PMID 16584857.

9. Chiang LC, Ng LT, Lin IC, Kuo PL, Lin CC. Anti-proliferative effect of apigenin and its apoptotic induction in human Hep G2 cells. Cancer Lett. 2006;237(2):207-14. doi: 10.1016/j.canlet.2005.06.002, PMID 16023288.

10. Vargo MA, Voss OH, Poustka F, Cardounel AJ, Grotewold E, Doseff AI. Apigenin-induced apoptosis is mediated by the activation of PKCdelta and caspases in leukemia cells. Biochem Pharmacol. 2006;72(6):681-92. doi: 10.1016/j.bcp.2006.06.010, PMID 16844095.

11. Richardson KE, Tolbert NE. Oxidation of glyoxylic acid to oxalic acid by glycolic acid oxidase. J Biol Chem. 1961;236:1280-4. doi: 10.1016/S0021-9258(18)64165-1, PMID 13741299.

12. Sawaki S, Hattori N, Morikawa N, Yamada K. Oxidation and reduction of glyoxylate by lactate dehydrogenase. J Vitaminol. 1967;13(2):93-7. doi: 10.5925/jnsv1954.13.93.

13. Romano $M$, Cerra $M$. The action of crystalline lactate dehydrogenase from rabbit muscle on glyoxylate. Biochim Biophys Acta. 1969;177(3):421-6. doi: 10.1016/03044165(69)90304-3, PMID 4306839.

14. Sugiyama N, Taniguchi N. Evaluation of the role of lactate dehydrogenase in oxalate synthesis. Phytochemistry. 1997;44(4):571-4. doi: 10.1016/s0031-9422(96)00629-2, PMID 9041714.

15. Trease E, Evans W. Textbook of pharmacognosy. 3rd ed. London: Bailliere Tindal; 1989. p. 81-90.

16. Asker H, Davies D. Purification of rat liver enzymes involved in the oxidation of glyoxylate. Biochim Biophys Acta. 1983;761(1):103-8. doi: 10.1016/0304-4165(83)90367-7, PMID 6357283.

17. Liebler DC, Burr JA, Philips L, Ham AJ. Gas chromatographymass spectrometry analysis of vitamin $\mathrm{E}$ and its oxidation products. Anal Biochem. 1996;236(1):27-34. doi: 10.1006/abio.1996.0127, PMID 8619492.

18. Aysal P, Ambrus A, Lehotay SJ, Cannavan A. Validation of an efficient method for the determination of pesticide residues in fruits and vegetables using ethyl acetate for extraction. J Environ Sci Health B. 2007;42(5):481-90. doi: $10.1080 / 19312450701392490$, PMID 17562455.

19. Ibrahim M, Aal MAE. Spectroscopic study of the interaction of heavy metals with organic acids. Int J Environ Pollut. 2008;35(1). doi: 10.1504/IJEP.2008.021134.

20. Ibrahim M, Hameed AJ, Jalbout A. Molecular spectroscopic study of River Nile sediment in the Greater Cairo region. Appl $\begin{array}{lll}\text { Spectrosc. } & 2008 ; 62(3): 306-11 .\end{array}$ 10.1366/000370208783759795, PMID 18339239.

21. Subramnian S, Nagarjan S. Flavonoids of the seeds of Crotolaria retusa and Crotolaria striata. Curr Sci (India). 1969;3:65.

22. Kong LD, Zhang $Y$, Pan X, Tan RX, Cheng CHK. Inhibition of xanthine oxidase by liquiritigenin and isoliquiritigenin isolated from Sinofranchetia chinensis. Cell Mol Life Sci. 2000;57(3):500-5. doi: 10.1007/PL00000710, PMID 10823249.

23. Nisha CM, Kumar A, Vimal A, Bai BM, Pal D, Kumar A. Docking and ADMET prediction of few GSK-3 inhibitors divulges 6bromoindirubin-3-oxime as a potential inhibitor. J Mol Graph Model. 2016;65:100-7. doi: 10.1016/j.jmgm.2016.03.001, PMID 26967552. 
24. Gu R, Wang Y, Long B, Kennelly E, Wu S, Liu B, Li P, Long C. Prospecting for bioactive constituents from traditional medicinal plants through ethnobotanical approaches. Biol Pharm Bull. 2014;37(6):903-15. doi: 10.1248/bpb.b14-00084, PMID 24882403.

25. Segel IH. Biochemical calculations. 2nd ed. New York: John Wiley \& Sons; ISBN: 978-0-471-77421-1; 1976.

26. Reddy UDC, Chawla AS, Deepak M, Singh D, Handa SS. High pressure liquid chromatographic determination of bergenin and (+) -afzelechin from different parts of Paashaanbhed (Bergenia ligulata yeo). Phytochem Anal. 1999;10(1):44-7. doi: 10.1002/(SICI)1099-1565(199901/02)10:1<44::AID-

PCA424>3.0.CO;2-4.
27. Vaishali A, Vikas M, Krishnapriya M, Sanjeevani G. Identification of potential antioxidants by in vitro activity guided fractionation of Bergenia ligulata. Pharmacogn Magzine. 2008;4(15):78-84.

28. Kirtikar K, Basu B. Indian medicinal plants. Vol. II. Periodical exports, D-42, Vivek vihar, Delhi, India; 1975.

29. Bahl C, Murari R, Parthasarathy M, Seshadri T. Components of Bergenia strecheyi and Bergenia ligulata. Indian J Chem. 1974;12:1038-9.

30. Manandhar N. Plants and people of Nepal. Oregon: Timber Press; 2002. 